\title{
Oxygen Generating Polymeric Nano Fibers That Stimulate Angiogenesis and Show Efficient Wound Healing in a Diabetic Wound Model
}

This article was published in the following Dove Press journal: International Journal of Nanomedicine

\author{
Mubashra Zehra' \\ Waliya Zubairi ${ }^{2}$ \\ Anwarul Hasan (1D ${ }^{3,4}$ \\ Hira Butt' \\ Amna Ramzan' \\ Maryam Azam² \\ Azra Mehmood' \\ Mojtaba Falahati ${ }^{5}$ \\ Aqif Anwar Chaudhry ${ }^{2}$ \\ Ihtesham Ur Rehman $\mathbb{D D}^{6}$ \\ Muhammad Yar $^{2}$ \\ 'National Center of Excellence in \\ Molecular Biology (CEMB), University of \\ the Punjab, Lahore 53700, Pakistan; \\ ${ }^{2}$ Interdisciplinary Research Centre in \\ Biomedical Materials, COMSATS \\ University Islamabad, Lahore Campus, \\ Lahore 54000, Pakistan; ${ }^{3}$ Department of \\ Mechanical and Industrial Engineering. \\ College of Engineering, Qatar University, \\ Doha 27I3, Qatar; ${ }^{4}$ Biomedical Research \\ Center, Qatar University, Doha 2713 , \\ Qatar; ${ }^{5}$ Department of Nanotechnology, \\ Faculty of Advanced Sciences and \\ Technology, Pharmaceutical Sciences \\ Branch, Islamic Azad University, Tehran, \\ Iran; ${ }^{6}$ Engineering Department, Faculty of \\ Science and Technology, Lancaster \\ University, Lancaster LAI 4YW, UK
}

Correspondence: Anwarul Hasan;

Muhammad Yar

Tel +974-70569169; +92 42001007 Ext 828

Email ahasan@qu.edu.qa;

drmyar@cuilahore.edu.pk
Introduction: Diabetic wounds are challenging to treat due to a wide range of pathophysiological changes. Hypoxia is one of the predominant contributing factors of poor vascularization and chronicity in diabetic wounds. This study was designed to develop polycaprolactone (PCL)-based oxygen-releasing electrospun wound dressings and evaluate their efficacy for improved full thickness wound healing in diabetic rats.

Methods: PCL-based oxygen releasing wound dressings were made using electrospinning technology. The developed dressings were characterized in terms of physical as well as biological properties both in vitro and in vivo. E-spun nanofibrous dressings were physically characterized with scanning electron microscopy, Fourier-transform infrared spectroscopy, and Energy-dispersive X-ray spectroscopy. To study the likely impact of the fabricated wound dressings in hypoxic conditions, HIF-1 $\alpha$ expression analysis was carried out both at gene and protein levels. Wound dressings were further evaluated for their healing potential for extensive wounds in diabetic rat models.

Results: The experimental results showed that the developed dressings were capable of continuously generating oxygen for up to 10 days. Cell studies further confirmed pronounced expression of HIF-1 $\alpha$ at gene and protein levels in cells seeded on PCL-sodium percarbonate (SPC) and PCL scaffolds compared with the cells cultured on a tissue culture plate. Chorioallantoic membrane assay revealed the supportive role of oxygen releasing dressings on angiogenesis compared to the control group. Histological assessment of the regenerated skin tissues proved that full thickness wounds covered with SPC loaded PCL dressings had a comparatively better vascularized and compact extracellular matrix with completely covered thick epithelium.

Discussion: The developed oxygen generating polymeric nanofibrous wound dressings could potentially be used as an envisioned approach for the efficient recovery of chronic diabetic wounds.

Keywords: skin regeneration, diabetic wound healing, oxygenation, polymeric nanofibers, electrospinning

\section{Introduction}

Diabetes is a growing major health concern affecting millions of people all over the world. ${ }^{1}$ Diabetic patients have about $15-25 \%$ chance of developing chronic wounds, ie, foot, venous, and pressure ulcers during their lifetime. ${ }^{2}$ All diabetic ulcers are classified as non-healing chronic wounds that produce skin interruption with distressed and extended healing process. Among all, diabetic foot ulcers (DFUs) have a global prevalence of $6.3 \%$, with increased threats of lower limb 
amputation due to high risks of wound infection resulting from sepsis. This creates a heavy burden on public health globally. ${ }^{3}$ Overall, various studies reported that ulcers are leading cause of $85 \%$ of the amputations and this create the up to $50 \%$ risks of ulcer re-occurrence at a collateral wound site. ${ }^{4,5}$

With the increasing incidence of diabetes around the globe, understanding the mechanism in charge of poor diabetic wound healing has become a critical medical problem. It is established that more than 100 known pathophysiological changes impede wound healing in patients with diabetes. ${ }^{6}$ In addition, the prolonged hypoxic environment due to damaged blood vascular supply in diabetic wounds leads to chronicity and delayed healing response. $^{7}$ Proper oxygen supply is indeed required for normal wound healing as it regulates the cell proliferation, migration and angiogenesis with enhancement of collagen deposition and epithelization of regenerative tissue. ${ }^{8}$ Hypoxia-inducible factor-1 (HIF-1), being one of the main controllers of oxygen homeostasis, regulates the healing outcomes. HIF-1 deficiency due to prolonged hypoxia causes the formation of non-healing ulcers. Similarly, HIF-1 stabilization during early hypoxia will contribute to normal wound recovery through its role in cell survival, cell migration, release of growth factors, and matrix synthesis during the course of the healing process. ${ }^{9,10}$ Thus, hypoxia presence is an important predictive determinant of wound repair and can be considered as the best target to cure diabetic wounds.

Oxygen releasing biomaterials are a considerably emerging preference in the tissue engineering field that aims to produce and supply oxygen at the injured place, thus supporting effective wound recovery. ${ }^{11}$

Several advanced treatments, such as the skin derived from bioengineered silver alginate dressings and super absorbents, are currently available on the market. ${ }^{12}$ These treatments are used for various injuries, including diabetic wounds ${ }^{12}$ or deep burn injuries. ${ }^{13}$ For this purpose, some strategies contribute to the production of a potential bandage which can be used as a promising platform to heal damaged tissues. Modified fibers can be used as outstanding scaffolds in controlling drug/oxygen release over a long time period, which can be tuned by physicochemical structure of nanofiber.

It has been shown that nanomedicine in combination of some hormone or oxygen producing agents can improve diabetic wound healing. ${ }^{14}$ Also, oxygen carrying nanocomposites can improve the recovery rate of chronic diabetic wounds by supplying the oxygen upon external stimulation. ${ }^{15}$ By creating similar environments as those of body tissues, 3D nano scaffolds offer a new perspective in cell culture, drug delivery, and tissue repair. ${ }^{16,18}$

Poly caprolactone (PCL), being a biocompatible, hydrophobic, and non-toxic polymer, is widely used as a drug carrier to prepare implantation devices and biomedical grafts in the tissue engineering field. ${ }^{19,20}$ Many oxygen releasing agents have been tested for their potential in improving diabetic wounds but oxygen releasing biomaterials, especially peroxide based biopolymers, are of special interest for their tunable properties in terms of controlled oxygen generation at the wound site. Sodium per carbonate (SPC) is one of the potent peroxide-based oxygen generating materials that has been found effective for treatment of dermal wounds. SPC is a moderately water soluble salt $\left(<0.01 \%\right.$ at $\left.20^{\circ} \mathrm{C}\right)$ that releases hydrogen peroxide and ultimately oxygen on decomposition. ${ }^{11}$ To our knowledge, no one has investigated its effectiveness for the treatment of diabetic wound models yet, so this study was aimed to explore therapeutic features of SPC for diabetic wound applications.

In the present study, we have developed PCL polymer matrix based electrospun nanofibrous wound dressings loaded with inorganic SPC salt that can chemically generate oxygen in-situ. This study includes the assessment of sustained oxygen release from prepared dressings for a minimum of 4 days, followed by evaluation of their efficacy through in-vitro cell studies, and a CAM assay to evaluate their angiogenesis potential and healing response of a full-thickness chronic diabetic wound rat model.

\section{Materials and Methods Materials}

PCL molecular weight $80 \mathrm{kDa}$ (Sigma Aldrich, St Louis, MO) was the basic polymer used. Sodium per carbonate (Sigma Aldrich) was used as the oxygen generating source material. The reagents used include dichloromethane (Scharlu), dimethylformimide, tetrahydrofuran (Fisher Scientific, Hampton, NH) and PBS tablets (7.4 pH) (Daejung).

\section{Preparation of Oxygen Generating PCL Electrospun Mats}

A 7\% solution of PCL was prepared in dichloromethane: dimethylformimide (5:1.25) and divided into two equal parts, the first a blank solution used as standard (PCL- 
Control) and the second part had $2 \%$ of sodium percarbonate (PCL-SPC). SPC solution was sonicated for uniform dispersion of particles. Electrospun sheets from each solution were prepared by applying a voltage of $19 \mathrm{kV}$ at a flow rate of 50 $\mu \mathrm{L}$ at room temperature. Solutions were stirred for 3 hours prior to fabrication. Prepared electrospun fiber mats were dried and kept under vacuum. Oxygen release was measured by hydroxyl ion formation and co-related with oxygen production. For this, film specimens of $1 \times 1 \mathrm{~cm}$ dimension were cut and added in PBS solution of $\mathrm{pH}$ 7.0. The increase in $\mathrm{pH}$ was monitored by a $\mathrm{pH}-\mathrm{meter}$ for 3-10 days.

\section{SEM and Quantification of Fiber Diameter}

SEM was performed to study the architectural details of the fabricated electrospun mats. Samples were coated with gold and images were acquired at $20 \mathrm{kx}$ and $5 \mathrm{kx}$ magnifications. The surface morphology of electrospun fiber sheets was observed by a scanning electron microscope (Model JEOLJSM 6480). Samples were coated with a gold layer prior to analysis at different magnifications . Fiber diameter was calculated using image $\mathrm{J}$ software.

\section{FTIR Analysis}

Fourier transform infrared spectroscopy [(FTIR), Thermo Nicolet, USA] was used to evaluate the chemical structural properties of all prepared samples. Spectra were obtained over a range of $4000-400 \mathrm{~cm}^{-1}$ at $8 \mathrm{~cm}^{-1}$ resolution at room temperature by accumulation of 128 scans.

\section{Oxygen Releasing Assay}

Oxygen generating electrospun fiber mats were fabricated and evaluated for their oxygen releasing capacity in-vitro. The fabricated-spun sheets were immersed in water and the appearance of tiny bubbles on the sheet surface due to release of oxygen gas was observed. To calculate the time duration, fiber sheets having SPC can generate oxygen for, concentration of dissolved oxygen within the media was quantified up to 10 days.

\section{HIF I $\alpha$ Gene and Protein Expression Analysis -invitro Culturing of Human Skin Fibroblasts}

All studies involving collection and processing of human skin tissues were carried out after getting approval from a formally constituted Institutional Review Board (IRB), National Centre of Excellence in
Molecular Biology, Lahore, Pakistan, in accordance with the Declaration of Helsinki for Human Research. Human skin biopsies were obtained from the volunteer donors with written informed donor consent. Fibroblasts were isolated and cultured following a procedure reported in our previous study. ${ }^{21}$ Scaffolds were sterilized by dipping in absolute ethanol for 30 seconds followed by washing with sterile PBS three times (2 minutes each). Sterile electrospun mats were seeded with fibroblasts at passage 3 and culture maintained for 24 hours at $5 \% \mathrm{CO}_{2}$ at $37^{\circ} \mathrm{C}$ in an incubator.

\section{Semi-Quantitative Real Time PCR (qPCR)}

Quantitative expressional analysis of the HIF1 $\alpha$ gene in experimental groups including cell seeded scaffolds as well as a control group, ie, cells cultured directly on a tissue culture plate was done by qPCR. The experiment was performed as per manufacturer's protocol using Maxima SYBR Green qPCR Master Mix (Fermentas, USA) and run on PikoReal 96 real time PCR (Thermo Scientific, USA). Analysis of relative gene expression was done using quantification cycle (cq) values. GAPDH was used for normalization.

\section{Western Blotting}

HIF1 $\alpha$ protein expression was studied through Western blotting. Briefly, extracted proteins from all groups were size separated through SDS-PAGE followed by transfer on to the nitrocellulose membrane using a semi-dry tansblot system (Bio-Rad) and stained with primary HIF1 $\alpha$ antibody (Abcam, USA). The final blot was developed with 5bromo-4-chloro-3-indolyl phosphate/nitro blue tetrazolium (NBT/BCIP) substrate (Sigma Aldrich). Densitometric analysis was done with Image $\mathrm{J}$ software.

\section{Chorioallantoic Membrane (CAM) Assay}

Fertilized eggs were purchased from Blue Bird (Lahore, Pakistan). On day 7 a small window of $1 \times 1 \mathrm{~cm}$ was made on egg shell and the oxygen generating electrospun mats were placed inside the eggs (one sample per egg) through the created window. They were sealed by paraffin film followed by reincubation in a 55\% humified incubator at $37^{\circ} \mathrm{C}$ for chick growth. All eggs were sacrificed on day 14 and comparative angiogenic analysis of both PCL control as well as PCL-SPC group was done by taking images with a light microscope (Mitotic, China). 


\section{In-vivo Evaluation in Diabetic Rat Model Induction of Diabetes}

The in-vivo study was approved by the institutional Ethics Committee for Animal Care, National Centre of Excellence in Molecular Biology, Lahore, Pakistan. Diabetes was induced in 12 male Sprague Dawley (SD) rats weighing $250-300 \mathrm{~g}$, by intraperitoneal administration of streptozotocin (STZ) drug $(40 \mathrm{mg} / \mathrm{kg}$ of the body weight). ${ }^{22}$ Blood glucose level was monitored using Accu Chek Active plus, every 72 hours after administration of first dose of STZ drug using rats' tail blood for 12 days (see Supplementary Data). Rats with $>300 \mathrm{mg} / \mathrm{dL}$ glucose were considered as diabetic.

\section{Implantation of Mats on Diabetic Wound Models}

Rats with successful induction of diabetes were divided into three experimental groups $(\mathrm{n}=3)$, ie, Control, $\mathrm{PCL}$ control, and PCL-SPC. All surgical procedures involved in the animal study had been performed after getting them approved from the Institutional Ethics Committee for Animal Care, National Centre of Excellence in Molecular Biology (CEMB). Prior to surgery, intraperitoneal anesthesia [ketamine $(87 \mathrm{mg} / \mathrm{kg})$ and xylazine $(14 \mathrm{mg} / \mathrm{kg})]$ was given to all rats. The dorsal surface of the rats was shaved with a hair trimmer (Panasonic Model) and sterilized with 70\% ethanol spray. Then $2 \times 2 \mathrm{~cm}^{2}$ full thickness excisional wounds (one wound/rat) were created on the proximal end of medial dorsal plane followed by quick implantation of electrospun fibrous mats. Silicon splints were fixed around the periphery of the created wounds and wounds were covered with Mepore ${ }^{\mathrm{TM}}$ bandage for 3 days. Control rats $(n=3)$ underwent sham excisional surgery without implantation of any biodegradable membrane. All experimental animals had easy and free access to food and water.

\section{Assessment of Wound Closure (\%)}

Wound closure (\%) was calculated on days 3, 7, 14, 21, and 27 after implantation of the mats, using a given formula $^{23}$ by using image $J$ software (Image $J$ version 1.52a). For this purpose, high resolution images of wounds of all rats were captured at a uniform magnification.

$$
\begin{aligned}
& \% \text { of Wound } \\
& \text { Closure }
\end{aligned}=\frac{\begin{array}{l}
\text { Wound Area } \\
\text { on Day } 0
\end{array}-\begin{array}{l}
\text { Open } \\
\text { Wound Area }
\end{array}}{\text { Wound Area on Day } 0} \times 100
$$

\section{Histology}

On complete wound closure, all rats were sacrificed using chloroform asphyxiation at day 30. Skin biopsies were collected by excising the re-epithelialized, newly-formed skin tissue and fixed in 10\% neutral buffered saline overnight. The next day, skin tissues were processed in accordance with standard tissue processing protocol and embedded in paraffin wax; $5 \mu \mathrm{m}$ thick sections were cut using Microm (HM-340E, Microm Inc. USA). Prepared sections were stained with hematoxylin and eosin (H\&E) stains for histology.

\section{Statistical Analysis}

All obtained data was analyzed thoroughly by Graph Pad Prism-5 software (USA). ANOVA was performed with Bonferroni post-test for multiple comparisons between individual groups. Student's t-tests were applied to compare the in-vitro cell culture results and amount of oxygen released from different prepared samples. Densitometric analysis was done with image $\mathrm{J}$ software. All experiments were performed in triplicate using three different cell cultures and tissue samples from at least three rats per experimental group.

\section{Results}

In this study, prepared oxygen generating PCL electrospun was characterized by different techniques followed by some in-vitro and in-vivo studies. In-vitro studies were done on human skin fibroblasts to assay the HIF1 $\alpha$ mRNA and protein expression, Afterwards, CAM assay was done to carry out the angiogenic analysis of both the PCL control and PCL-SPC groups In-vivo investigations were done on diabetic wound models to assess the wound closure and histopathological changes after treatment with fabricated mats.

\section{Fiber Diameter}

Figure 1A shows the electrospun fibers of PCL-Control and PCL-SPC. SEM images showed the random alignment of fibers, as their collection was made on the static collector plate. Uniform interconnectivity of elongated thin fibers arranged in a crisscross manner with pore in the polymer matrix were observed that confirmed more surface area and porosity among fibers.

The PCL fibers without sodium per carbonate (SPC) were considered control samples. The fiber diameter of each sample was measured by using image $J$ software. 
A

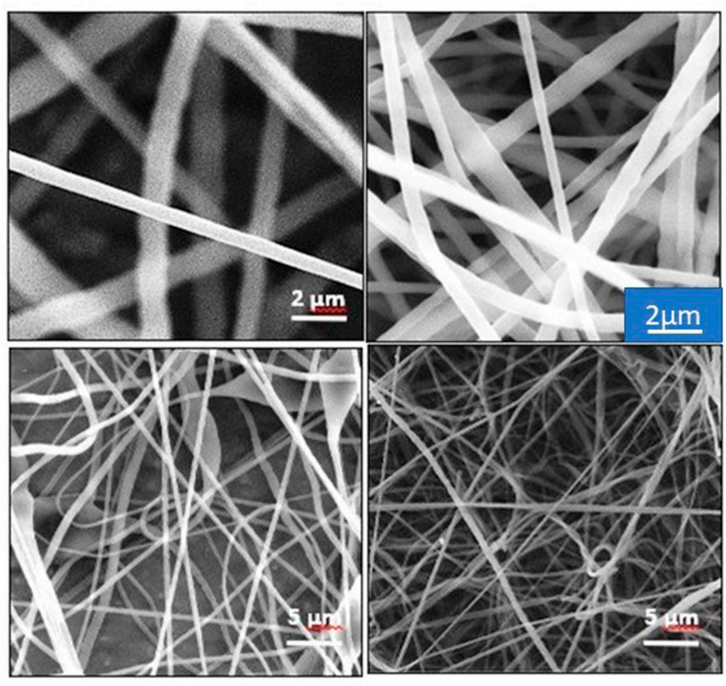

B

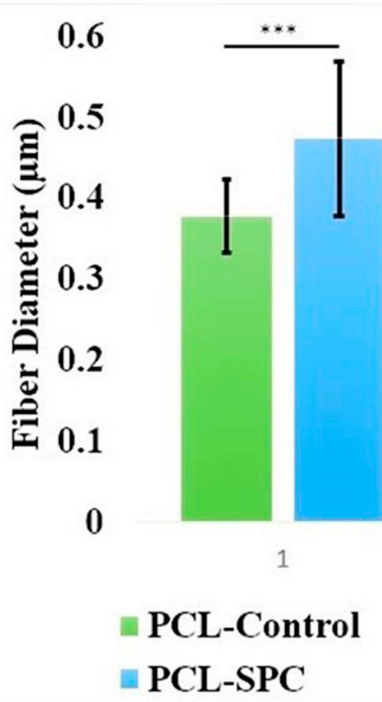

Figure I Scanning electron microscope (SEM) analysis. (A) SEM images of PCL-Control and PCL-SPC at 5 and 20 kx magnifications. (B) Comparative study of fiber diameter of synthesized electrospun mats. Data are presented as mean \pm SD.

The fibers having SPC in their polymer matrix had a greater fiber diameter $(0.473 \pm 0.9 \mu \mathrm{m})$ than that of the control ones $(0.377 \pm 0.45 \mu \mathrm{m})$. Figure $1 \mathrm{~B}$ shows comparative results of fabricated electrospun mats. The $t$-test showed a significant difference between fiber diameters of the same polymer's mats.

\section{FTIR and EDS Analysis}

The FTIR spectra of PCL and PCL-SPC are shown in Figure 2A. The characteristic peaks for PCL are: C-H stretching vibrations are observed at $2,942 \mathrm{~cm}^{-1}$ and $2,866 \mathrm{~cm}^{-1}$, while the peaks ranging from 1,471-1,418 $\mathrm{cm}^{-1}$ represent $-\mathrm{CH}_{2}$ bend vibrations. Peak for carbonyl group $(\mathrm{C}=\mathrm{O})$ is visible at $1,724 \mathrm{~cm}^{-1}$ wavelength which shows its characteristic stretching vibration. C-O bond stretching vibration peak for PCL is observed at 1,250 $1,100 \mathrm{~cm}^{-1}$. SPC in FTIR spectra shows the presence of carbonyl $(\mathrm{C}=\mathrm{O})$ and $\mathrm{C}-\mathrm{O}$ bond. PCL-SPC scaffold FTIR spectra shows the sharp and high intensity peak of carbonyl group at $1,724 \mathrm{~cm}^{-1}$ as intensity of the carbonyl group is increased with the addition of sodium per carbonate in polymer matrix. The intensity of the $\mathrm{C}-\mathrm{O}$ peak at 1,250 $1,100 \mathrm{~cm}^{-1}$ and $\mathrm{C}-\mathrm{O}-\mathrm{C}$ bond at $1,060-1,100 \mathrm{~cm}^{-1}$ stretch in PCL-SPC spectra is also increased, confirming the presence of SPC in the PCL polymer matrix.

EDX spectroscopy is the precise tool to detect the elements in the sample and applied with SEM for composition confirmation. Figure 2B illustrates the EDS spectra for synthesized electrospun mats $(n=6)$. PCL-Control spectra included the peaks characteristic for the presence of carbon and oxygen elements only, basic elements of an organic compound, confirming the purity of electrospun mats. In addition to the increased intensity of carbon and oxygen representative peaks, EDS spectra of PCL-SPC confirmed the presence of sodium in each spectrum. By these observations, it was evident that synthesized electrospun mats had sodium per carbonated impregnated in their polymer matrix.

\section{Evaluation of Oxygen Release In-vitro}

Throughout the experiment, a relatively uniform elevation of oxygen level was observed in the solution with $\mathrm{pH}$ between 7.3 and 7.6 (Figure 3). However, no increase in $\mathrm{pH}$ or release of oxygen was observed by PCL only fibers (control group) up to 10 hours, contrary to the PCL-SPC blend nanofibers which showed elevated $\mathrm{pH}$ of the solution efficiently over time. Slow release of oxygen out of the PCL fibers might be due to the very slow degradation characteristic of PCL.

\section{In-vitro Expression Analysis at Gene and Protein Levels}

The results in Figure 4A revealed that cells seeded on scaffolds showed significantly increased expression of HIF-1 $\alpha$ gene as compared to cells cultured directly on tissue culture plates without any scaffold (Control). The 
A

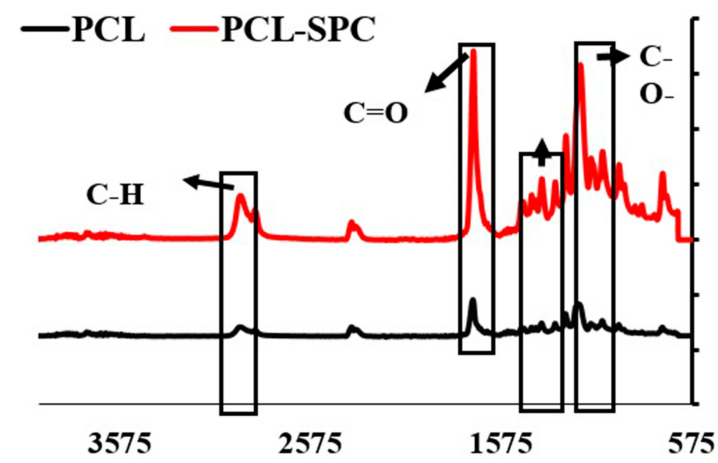

B
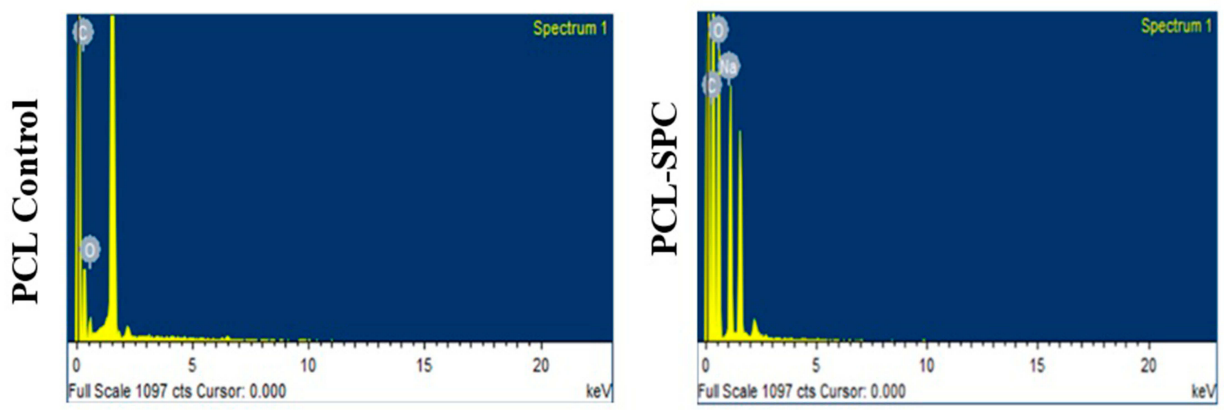

Figure 2 FTIR and EDS analyses of nanofibrous wound dressings. (A) FTIR analysis of PCL-SPC and PCL-Control samples. The vibrations at I,724 $\mathrm{cm}^{-1}$ and I,47I-I,4I8 $\mathrm{cm}^{-1}$ represent $\mathrm{C}=\mathrm{O}$ and $-\mathrm{CH}_{2}$ stretches, respectively, arrows indicate various functional groups. (B) EDS analysis of PCL-SPC and PCL Control samples. PCL-Control spectrum showed peaks characteristic for the existence of carbon and oxygen elements only, confirming purity of the samples. The presence of sodium was confirmed by enhanced intensity of carbon and oxygen representative peaks.

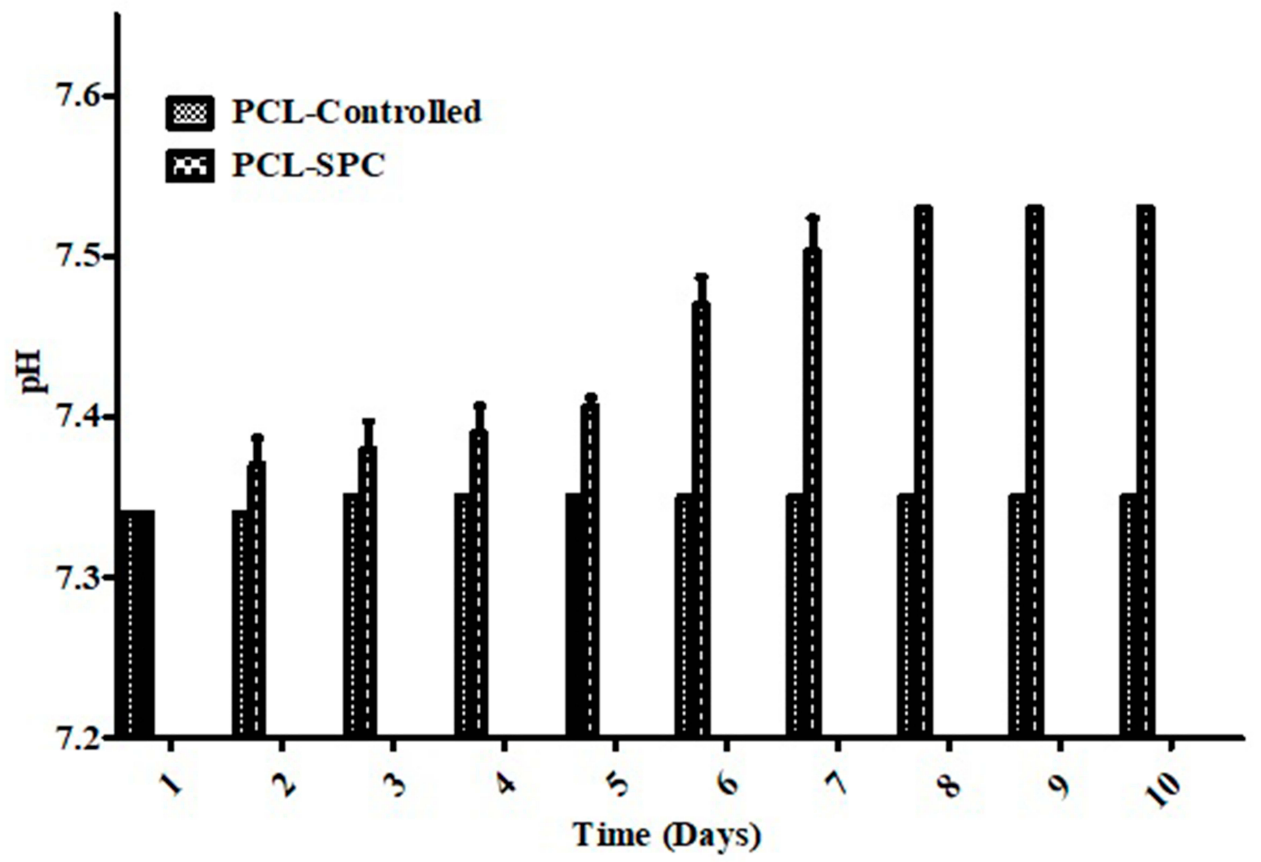

Figure 3 Oxygen release profile of electrospun mats over a time duration of 10 days in both PCL-Control and PCL-SPC groups. Data is presented as mean \pm SD. A relatively uniform enhancement of oxygen level was determined in the solution with PH between 7.3 and 7.6 in PCL-SPC blend nanofibers compared to PCL only fibers (control group). 

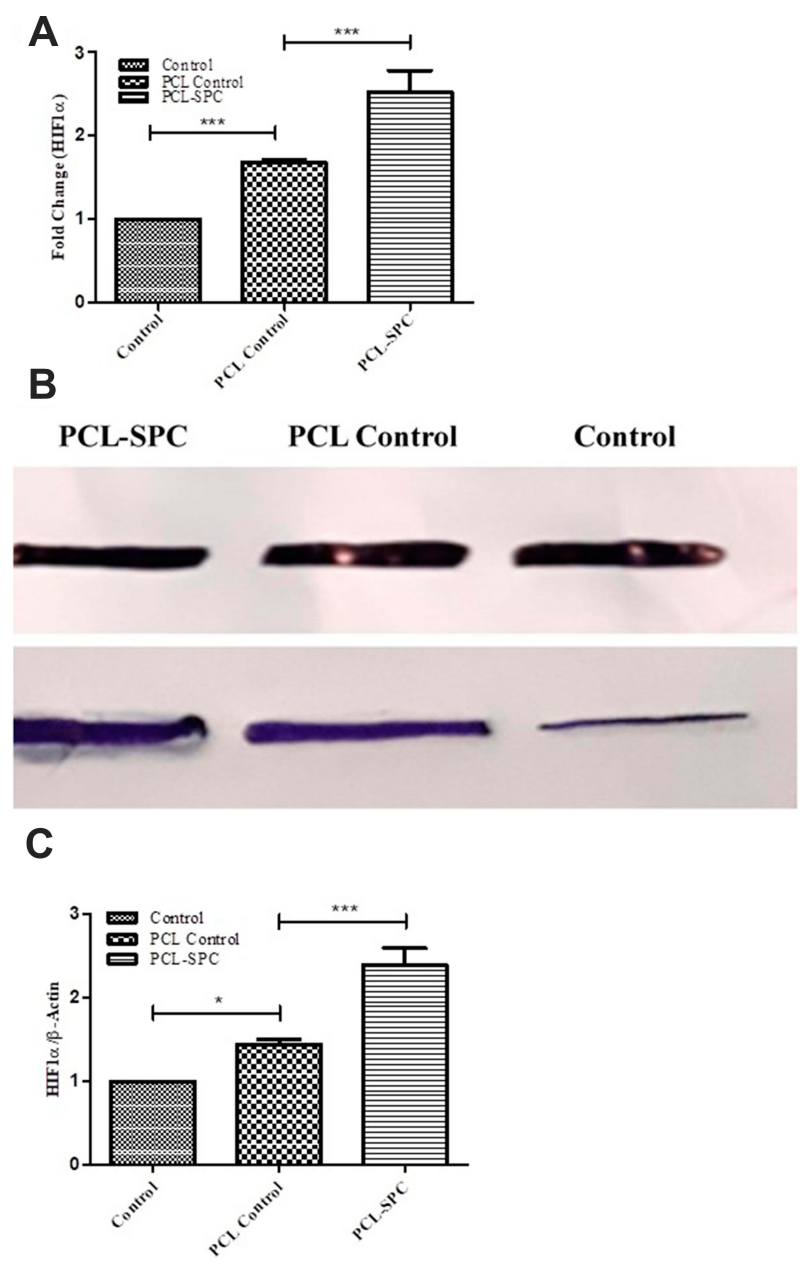

Figure 4 Molecular analysis of Hypoxia-inducible factor-I (HIF-I $\alpha$ ). (A) Relative mRNA expression analysis of HIF-I $\alpha$ gene in PCL-SPC; PCL Control and Control groups by q-PCR. (B-C) Protein expression analysis of HIF-I $\alpha$ protein in PCL-SPC; $\mathrm{PCL}$ Control and Control groups by Western blotting. Data are expressed as mean \pm SD. $* P \leq 0.05 ; * * * \leq 0.001$.

expression of HIF-1 $\alpha$ was found to be remarkably upregulated in the PCL-SPC group (2.52 \pm 0.26 -fold) as compared to the PCL Control group (1.68 \pm 0.03 -fold).

Findings of the HIF-1 $\alpha$ gene expression analysis were further confirmed by proteomic analysis of the same gene in various groups of cells seeded on the mats. Figures 4B and $\mathrm{C}$ illustrate the relative expression of HIF-1 $\alpha$ protein was significantly up-regulated in PCL-SPC $(2.39 \pm 0.3-$ fold) compared with the PCL Control group (1.44 \pm 0.06 fold).

\section{Chorioallantoic Membrane (CAM) Assay}

CAM assay studies were designed to check the angiogenic potential of oxygen releasing electrospun mats. Figure 5A shows implantation of electrospun mats in the fertilized egg for CAM assay. A greater number of blood vessels were present around the oxygen releasing mats than the polymer-control mats, supporting the fact that release of oxygen triggers angiogenesis, resulting inincreased rapid blood vessels formation. PCL-SPC electrospun mats were completely surrounded and impregnated by the blood vessels from all sides. On the other hand PCL-Control electrospun mats were not covered by a significant number of blood vessels and were only visible on the membrane.

Figure 5B demonstrates the blind scoring of blood vessels. PCL-SPC shows a greater number of blood vessels than PCL-Control electrospun fibers. Student's $t$-test was applied and a statistically significant difference, ie, $P<0.0001$ was found between PCL-control with PCL-SPC fibers.

\section{In-vivo Evaluation \\ Diabetes Confirmation in Rats}

Diabetes was successfully induced in diabetic models as evidenced by elevated blood sugar level and visible symptoms like gradual weight loss, lethargy, decreased mobility within the cage, and excessive consumption of chow. Weight loss in all diabetic rats was consistent with elevated blood sugar level, hence proving the STZ induced damage to islets of Langerhans in the pancreas.

\section{Wound Closure Assessment}

Wound healing analysis in diabetic rats was done by macroscopic and percentage wound closure assessment by application of PCL-SPC electrospun mats. Figure 6 shows the descriptive macroscopic images of sham, PCL control, and PCL-SPC experimental group wounds at Day 0 (transplantation day), 3, 7, 14, 21, and 27 (day of biopsies collection). Wound areas were calculated by using image $\mathrm{J}$ analysis of wound images.

Figure 7 shows the percentage wound closure analysis in all experimental groups at day 3, 7, 14, 21, and 27 posttransplantation. No significant difference was observed in all experimental groups throughout the whole duration.

However, H\&E staining (Figure 8) revealed that PCLSPC treatment was effective in improving the architecture of the epidermis and dermis comparative to both sham and PCL control groups indicative of better epithelization and wound recovery in the treatment group PCL-SPC than in the Control and PCL control groups.

\section{Discussion}

Nanofibers are a good option for wound dressing mats, given their biomimetic structure similarity with the 
A

Implanted Scaffolds Closer View
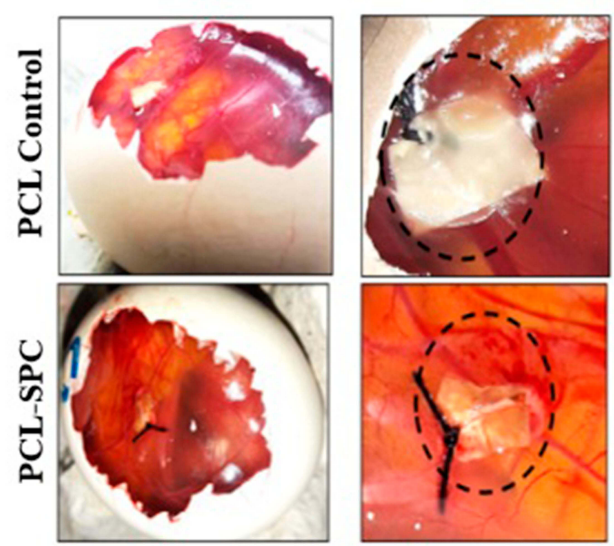

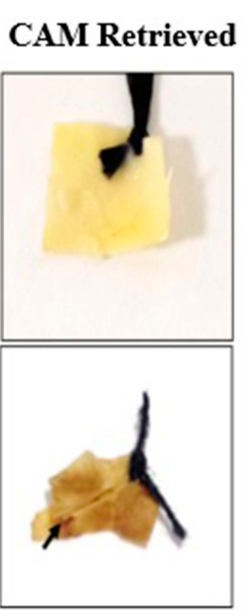

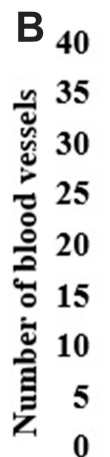

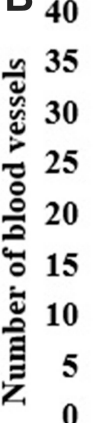

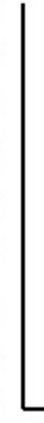

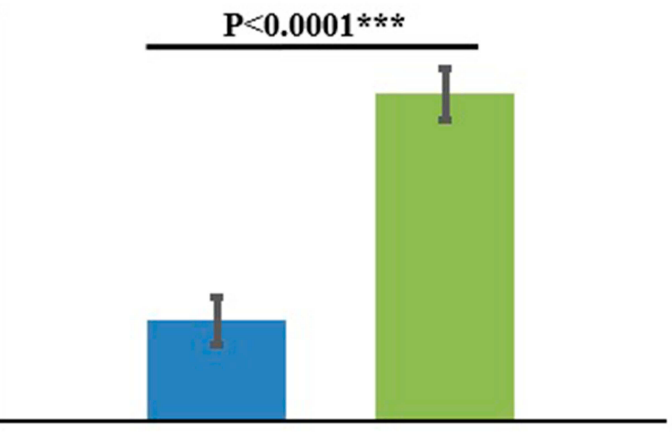

Angiogenesis

घCL-Control $\quad$ PCL-SPC

Figure 5 Chorioallantoic membrane (CAM) assay. (A) Electrospun mats implanted in the fertilized eggs. Closer images of the mats showed the presence of blood vessels around them. CAM assay retrieved samples are shown; arrows indicate the dried blood vessels. (B) Blood vessels quantification using images of the scaffolds retrieved from CAM assay by blind scoring. Data are presented as mean $\pm S D$. *** $\leq 0.0001$.

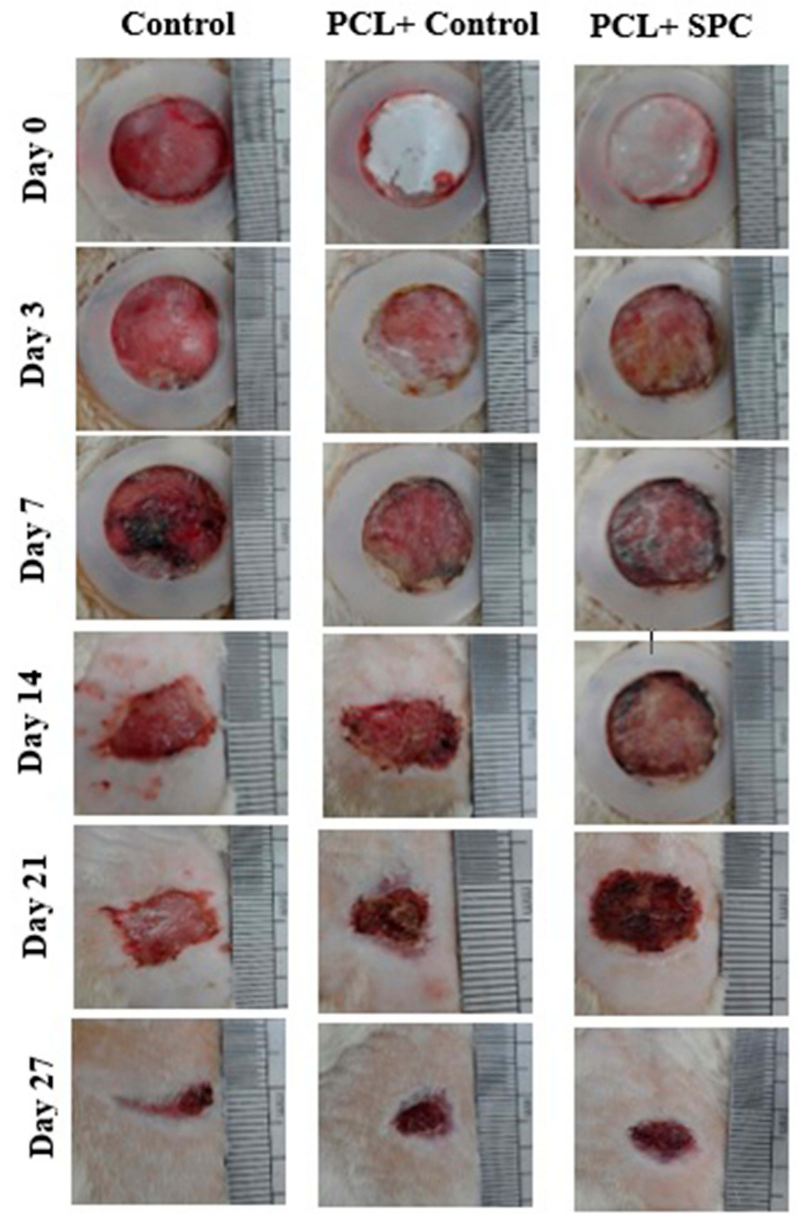

Figure 6 Representative macroscopic analysis of wounds. Sham, PCL-Control and PCL-SPC are shown at days 3, 7, 14, 21, and 27 post-wounding. Wound areas were calculated through image J software. No significant difference was observed in all experimental groups throughout the experimental study.

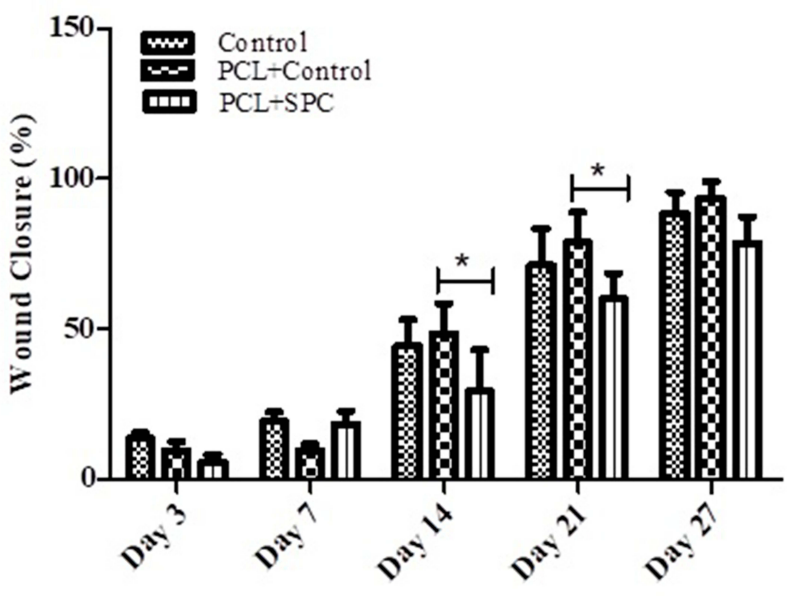

Figure 7 Percentage of wound size reduction. Graphical data are presented as mean $\pm S D(n=3)$ at days $3,7,14,21$, and 27 . ns, non-significant; $* P \leq 0.05$. No significant difference was reported in all experimental groups throughout the experimental study.

extracellular matrix (ECM) of tissues at nanometer scale. The structure of nanofibers with their unique properties increases their compatibility with the wound tissue and improves and accelerates wound healing. An ideal wound coating should be able to simulate the skin structure. Because of their physical properties, nanofibers can form a structure similar to the ECMs.

In diabetic patients, on the other hand, the wall of blood vessels is damaged after a prolonged duration. Forever, blockage of the veins decreases the blood supply to the organs, especially the lower limbs, and result in a 


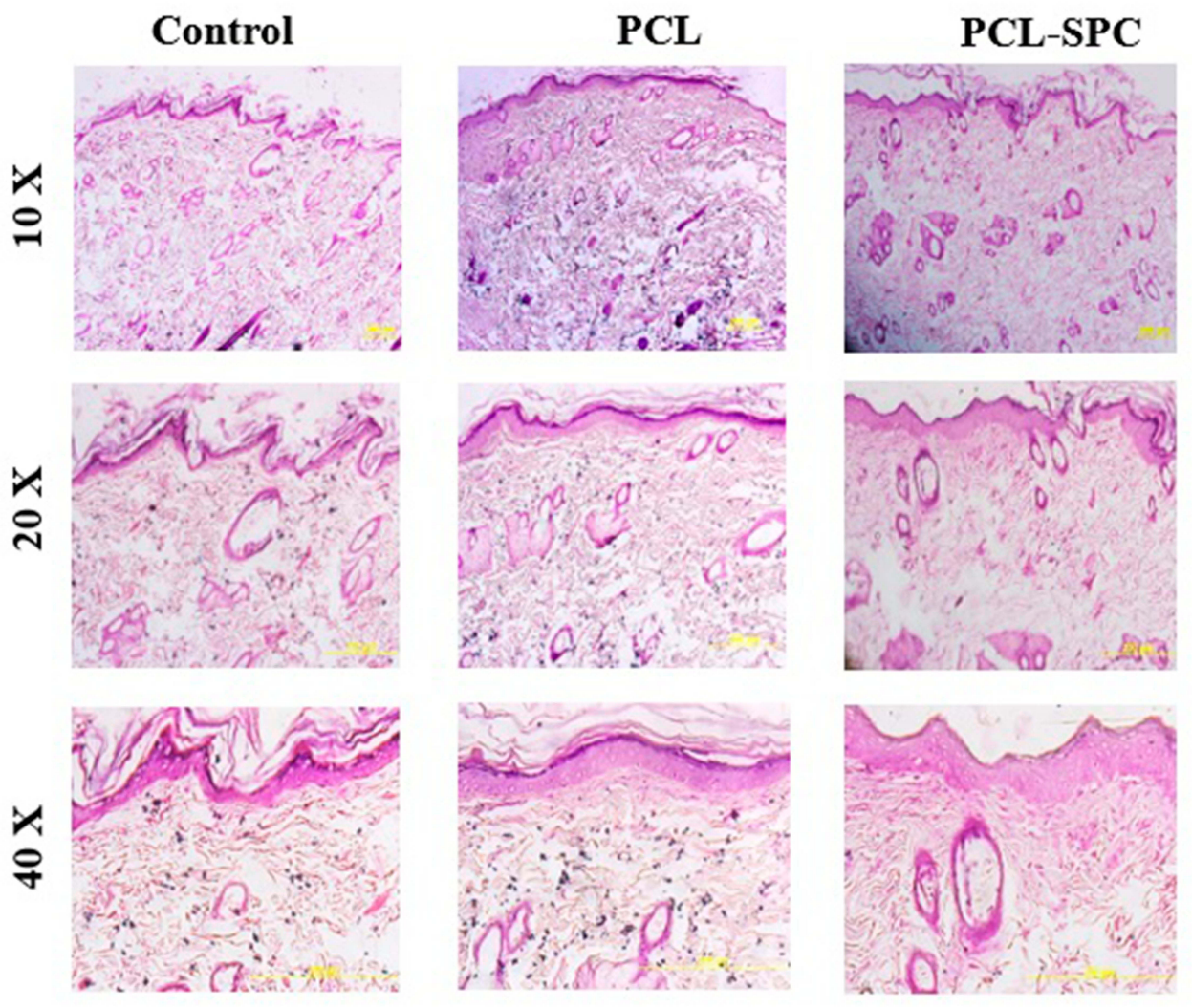

Figure 8 Representative images of H\&E stained healed skin sections harvested 27 days post-wounding. It can be observed that PCL-SPC treatment was promising in improving the architecture of the epidermis and dermis compared to both sham and PCL control groups.

mortality of the cells in the injured areas. ${ }^{18,24}$ Particularly, neuropathy caused by diabetes leads to a decrease in pain, even after serious damage to the surface of the skin, and eventually results in chronic ulcers. Despite the wide range of dressings, choosing apromising dressing is one of the major challenges for the development of diabetic wound healing. Many dressings designed for diabetic wound healing so far have either been removed from the pharmaceutical market due to serious problems or have not received public approval due to high costs and other medical conditions. A potential nano-scaffold must be both biocompatible and biodegradable, in addition to being able to mimic the biological microenvironment, both in terms of chemical composition and physical assembly. ${ }^{18,24,25}$ Also, a nano-scaffold should have the potential to be easy and convenient to use for the patient and ultimately low cost. Recently, nanofibers have received much attention in tissue engineering due to their structural nature. ${ }^{26}$ Indeed, their unique fibrous structures facilitate cellular function, proliferation, and infiltration. The current study approached the development of innovative electrospun oxygen generating wound dressings that comprised of
PCL polymer matrix providing chemical based oxygen from loaded inorganic salt (SPC) in a sustained manner. The most important characteristic of the developed dressings is that they can be prepared in a cost-effective manner and oxygen delivery can be tightly regulated through an optimized amount of SPC and by modifying the polymer composition. SEM analysis (Figure 1) performed to analyze the morphology of nanofibrous scaffolds showed that SPC loading increased the fiber diameter of fibrous scaffolds compared to control. Prepared electrospun scaffolds were characterized through FTIR and SEM analysis. FTIR spectra and EDS analysis (Figure 2) showed the interaction between PCL polymer matrix and SPC and confirmed that synthesized electrospun mats have sodium per carbonated impregnated in their polymer matrix. Prepared dressings are simple to use; and can release oxygen in a controlled way in-situ to the healing tissue for up to 10 days (Figure 3).

The oxygen released by the oxygen generating scaffolds has the ability to uphold cell viability inside the scaffold under hypoxic conditions. ${ }^{27}$ HIF- $1 \alpha$ is a vital transcription factor that facilitates cells' adaptation to 
low oxygen level and it is regulated specifically by hypoxic and hyperglycemic conditions, which are major factors of the diabetic complications. Many genes are regulated by HIF- $1 \alpha$ and above 90 genes are directly targeted by HIF-1 $\alpha$ through its functional interaction with hypoxia response elements. ${ }^{28,29}$ Additionally, these genes have been proved to be involved in angiogenesis, wound healing, cell proliferation, differentiation, apoptosis, and survival. $^{30,32}$ It has been studied that HIF- $1 \alpha$ enhances the survival of mesenchymal stem cells exposed to hypoxic condition. HIF- $1 \alpha$ has been shown to play a protective role against apoptosis of cardiac fibroblasts after hypoxic condition. ${ }^{33}$ Previous studies have proven that fibroblasts and endothelial cells when seeded on hydrogel nanofibrous scaffolds showed upregulated expression of HIF-1 $\alpha .{ }^{34}$ Our study also demonstrated that the expression of HIF-1 $\alpha$ was remarkably increased both at gene and protein levels in the PCL containing SPC group in cell studies (Figure 4).

Angiogenesis is an important factor for successful wound healing ${ }^{35}$ and Oxygen at an optimum concentration stimulates production of VEGF growth factor which is considered a most effective and long-term angiogenesis stimulator. $^{36,37}$ Findings of the CAM assay (Figure 5) confirmed that controlled supply of topical oxygen could induce blood vessel formation in-situ.

Compromised wound healing is a life-threatening problem along with prominent cause of non-traumatic lower extremities amputation in diabetic patients. ${ }^{38,39}$ The present study intended to investigate the healing effect of released oxygen from prepared dressings in a full thickness wounded diabetic rat model. Apparently, there was not any significant difference in wound closure duration between the sham and treated groups that could be attributed to use of silicone splints around the wound area to avoid skin contraction which is a common phenomenon in normal wound healing. But here it is important to consider the fact that in-situ oxygen release proved to improve re-epithelization and architecture of the epidermis and dermis in healed skin tissue of the PCL-SPC treated experimental group compared to the control groups, as evident by histological evaluation (Figure 8). Likewise, previous studies done by Chandra et al, ${ }^{11}$ showed that oxygen generating POG dressings resulted in better wound closure and faster re-epithelization in a pigs full-thickness wound healing model.

Ochoa $^{40}$ reported that laser-fabricated wound dressing patches can be used as a unique approach for promoting diabetic wound recovery through selective oxygenation. It has also been shown that increasing angiogenesis and oxygenation in a diabetic wound environment using smart polymeric hydrogels can be a promising strategy for promoting diabetic wound healing. ${ }^{41}$

This data may provide useful information regarding the production of some smart nanofibers that are specifically used to treat diabetic wounds. By applying oxygen-loading nanofibers, this dressing improves the wound healing efficiency compared to other dressings. Also, this product is relatively less expensive than the similar ones already reported due to its high production speed and reproducibility. Its industrial production can be performed with higher efficiency. The use of this dressing reduces the length of the treatment period, which, in addition to reducing the suffering of the users, will also reduce the cost of treatment. It should be noted that the innovative dressing should be improved into a transparent structure to easily monitor the condition of the wound and better manage the treatment process. Also, due to the nanocrystalline properties, this dressing can stay on the wound for a longer time duration while preventing the microorganisms from entering the wound and passing oxygen to the wound surface.

\section{Conclusion}

Accelerating the healing of any non-treatable chronic ulcer, such as diabetic and similar wounds that face limited and slow therapies can be of great interest. In this study, new and unique methods have been used to eventually lead to the healing of diabetic ulcers. Results indicated that the developed SPS-loaded PCL could increasingly produce oxygen for up to 10 hours, as evident by an efficient rise in $\mathrm{pH}$ of solution with time. Cellular assays also confirmed that the synthesized scaffold enhanced relative upregulation of HIF-1 $\alpha$ both in gene and protein levels as well as angiogenesis. Histological investigations showed that full thickness wounds dressed with SPC-loaded PCL agents provide a more promising vascularization and compact extracellular matrix. In conclusion, this strategy of topical supply of chemical-based oxygen showed angiogenesis stimulation in CAM assay and wound healing potential in diabetic wounded rat models. Thus, this offers a noteworthy approach to tackle the chronic hypoxia in hard to heal diabetic wounds and could be used clinically after particular testing in clinical settings.

\section{Acknowledgment}

We greatly acknowledge the funding support of the Higher Education Commission Pakistan Project No. 8762 and the grant NPRP9-144-03-021 and NPRP12S-0310-190276 by 
Qatar National Research Foundation a subsidiary of Qatar Foundation. All statements made here are sole responsibilities of the authors.

\section{Disclosure}

The authors report no conflicts of interest in this work.

\section{References}

1. Al-Lawati JA. Diabetes mellitus: a local and global public health emergency! Oman Med J. 2018;32(3):177-179. doi:10.5001/ omj.2017.34

2. Siddiqui AR, Bernstein JM. Chronic wound infection: facts and controversies. Clin Dermatol. 2010;28(5):519-526. doi:10.1016/j. clindermatol.2010.03.009

3. Zhang P, Lu J, Jing Y, et al. Global epidemiology of diabetic foot ulceration: a systematic review and meta-analysis. Ann Med. 2017;49 (2):106-116. doi:10.1080/07853890.2016.1231932

4. Moxey P, Gogalniceanu P, Hinchliffe R, et al. Lower extremity amputations - a review of global variability in incidence. Diabet Med. 2011;28(10):1144-1153. doi:10.1111/j.1464-5491.2011.03279.x

5. Gianino E, Miller C, Gilmore J. Smart wound dressings for diabetic chronic wounds. Bioengineering (Basel). 2018;5(3):51. doi:10.3390/ bioengineering5030051

6. Tsourdi E, Barthel A, Rietzsch H, et al. Current aspects in the pathophysiology and treatment of chronic wounds in diabetes mellitus. Biomed Res Int. 2013;2013:385641. doi:10.1155/2013/385641

7. Zhao R, Liang H, Clarke E, et al. Inflammation in chronic wounds. Int J Mol Sci. 2016;17(12):2085. doi:10.3390/ijms 17122085

8. Castilla DM, Liu ZJ, Velazquez OC. Oxygen: implications for wound healing. Adv Wound Care. 2012;1(6):225-230. doi:10.1089/wound.20 11.0319

9. Hong WX, Hu MS, Esquivel M, et al. The role of hypoxia-inducible factor in wound healing. Adv Wound Care (New Rochelle). 2014;3 (5):390-399. doi:10.1089/wound.2013.0520

10. Botusan IR, Sunkari VG, Savu O, et al. Stabilization of HIF-1 $\alpha$ is critical to improve wound healing in diabetic mice. Proc Natl Acad Sci U S A. 2008;105(49):19426-19431. doi:10.1073/pnas.0805230105

11. Chandra PK, Ross CL, Smith LC, et al. Peroxide-based oxygen generating topical wound dressing for enhancing healing of dermal wounds. Wound Repair Regen. 2015;23(6):830-841. doi:10.1111/wrr.12324

12. Jude E, Apelqvist J, Spraul M, et al. Prospective randomized controlled study of Hydrofiber ${ }^{\circledR}$ dressing containing ionic silver or calcium alginate dressings in non-ischaemic diabetic foot ulcers. Diabet Med. 2007;24(3):280-288. doi:10.1111/j.1464-5491.2007.02079.x

13. Opasanon S, Muangman P, Namviriyachote N. Clinical effectiveness of alginate silver dressing in outpatient management of partial-thickness burns. Int Wound J. 2010;7(6):467-471. doi:10.1111/j.1742481X.2010.00718.x

14. Desmet CM, Preat V, Gallez B. Nanomedicines and gene therapy for the delivery of growth factors to improve perfusion and oxygenation in wound healing. Adv Drug Deliv Rev. 2018;129:262-284. doi:10.1016/j.addr.2018.02.001

15. Wang S, Yin C, Han X, et al. Improved healing of diabetic foot ulcer upon oxygenation therapeutics through oxygen-loading nanoperfluorocarbon triggered by radial extracorporeal shock wave. Oxid Med Cell Longev. 2019;2019:1-10. doi:10.1155/2019/5738368

16. Grip J, Engstad RE, Skjæveland I, et al. Beta-glucan-loaded nanofiber dressing improves wound healing in diabetic mice. Eur J Pharm Sci. 2018;121:269-280. doi:10.1016/j.ejps.2018.05.031

17. Chao FC, Wu MH, Chen LC, et al. Preparation and characterization of chemically TEMPO-oxidized and mechanically disintegrated sacchachitin nanofibers (SCNF) for enhanced diabetic wound healing. Carbohydr Polym. 2020;229:115507. doi:10.1016/j.carbpol.2019.115507
18. Masood N, Ahmed R, Tariq M, et al. Silver nanoparticle impregnated chitosan-PEG hydrogel enhances wound healing in diabetes induced rabbits. Int J Pharm. 2019;559:23-36. doi:10.1016/j.ijpharm.2019.01.019

19. Ulery BD, Nair LS, Laurencin CT, et al. Biomedical applications of biodegradable polymers. J Polym Sci B Polym Phys. 2011;49 (12):832-864. doi:10.1002/polb.22259

20. Mondal D, Griffith M, Venkatraman SS, et al. Polycaprolactonebased biomaterials for tissue engineering and drug delivery: current scenario and challenges. Int J Polym Mater. 2016;65(5):255-265. doi:10.1080/00914037.2015.1103241

21. Butt H, Mehmood A, Ali M, et al. Protective role of vitamin E preconditioning of human dermal fibroblasts against thermal stress in vitro. Life Sci. 2017;184:1-9. doi:10.1016/j.lfs.2017.07.002

22. Leyva-García E, Lara-Martínez R, Morán-Zanabria L, et al. Novel insight into streptozotocin-induced diabetic rats from the protein misfolding perspective. Sci Rep. 2017;7(1):11552. doi:10.1038/s41598-017-11776-y

23. Azam M, Dikici S, Roman S, et al. Addition of 2-deoxy-d-ribose to clinically used alginate dressings stimulates angiogenesis and accelerates wound healing in diabetic rats. J Biomater Appl. 2019;34 (4):463-475. doi:10.1177/0885328219859991

24. Ahmed R, Tariq M, Ali I, et al. Novel electrospun chitosan/polyvinyl alcohol/zinc oxide nanofibrous mats with antibacterial and antioxidant properties for diabetic wound healing. Int J Biol Macromol. 2018;120(PtA):385-393. doi:10.1016/j.ijbiomac.2018.08.057

25. Augustine R, Hasan A, Patan NK, et al. Cerium oxide nanoparticle incorporated electrospun poly (3-hydroxybutyrate-co-3-hydroxyvalerate) membranes for diabetic wound healing applications. ACS Biomater Sci Eng. 2020;6(1):58-70. doi:10.1021/acsbiomaterials.8b01352

26. Hasan A, Morshed M, Memic A, et al. Nanoparticles in tissue engineering: applications, challenges and prospects. Int $J$ Nanomedicine. 2018;13:5637-5655. doi:10.2147/IJN.S153758

27. Shiekh PA, Singh A, Kumar A. Oxygen-releasing antioxidant cryogel scaffolds with sustained oxygen delivery for tissue engineering applications. ACS Appl Mater Interfaces. 2018;10(22):18458-18469. doi:10.1021/acsami.8b01736

28. Xiao $\mathrm{H}, \mathrm{Gu} \mathrm{Z}$, Wang $\mathrm{G}$, et al. The possible mechanisms underlying the impairment of HIF-1 $\alpha$ pathway signaling in hyperglycemia and the beneficial effects of certain therapies. Int J Med Sci. 2013;10 (10):1412-1421. doi:10.7150/ijms.5630

29. Cerychova R, Pavlinkova G. HIF-1, metabolism, and diabetes in the embryonic and adult heart. Front Endocrinol (Lausanne). 2018;9: 460. doi:10.3389/fendo.2018.00460

30. Shi YH, Fang WG. Hypoxia-inducible factor-1 in tumour angiogenesis. World J Gastroenterol. 2004;10(8):1082-1087. doi:10.3748/wjg. v10.i8.1082

31. Zimna A, Kurpisz M. Hypoxia-inducible factor-1 in physiological and pathophysiological angiogenesis: applications and therapies. Biomed Res Int. 2015;2015:549412. doi:10.1155/2015/549412

32. Hashimoto T, Shibasaki F. Hypoxia-inducible factor as an angiogenic master switch. Front Pediatr. 2015;3:33. doi:10.3389/fped.2015.00033

33. Du Y, Ge Y, Xu Z. Hypoxia-inducible factor 1 alpha (HIF-1 $\alpha$ )/ vascular endothelial growth factor (VEGF) pathway participates in angiogenesis of myocardial infarction in muscone-treated mice: preliminary study. Med Sci Monit. 2018;24:8870-8877. doi:10.12659/ MSM.912051

34. Chen H, Jia P, Kang H. Upregulating Hif-1 $\alpha$ by hydrogel nanofibrous scaffolds for rapidly recruiting angiogenesis relative cells in diabetic wound. Adv Healthc Mater. 2016;5(8):907-918. doi:10.1002/adhm.2015 01018

35. Honnegowda TM, Kumar P, Udupa E. Role of angiogenesis and angiogenic factors in acute and chronic wound healing. Plast Aesthet Res. 2015;2(4):243-249. doi:10.4103/2347-9264.165438

36. Shibuya M. Vascular endothelial growth factor (VEGF) and its receptor (VEGFR) signaling in angiogenesis: a crucial target for anti-and pro-angiogenic therapies. Genes Cancer. 2011;2(12):10971105. doi: $10.1177 / 1947601911423031$ 
37. Guo D, Wang Q, Li CY, et al. VEGF stimulated the angiogenesis by promoting the mitochondrial functions. Oncotarget. 2017;8 (44):77020-77027. doi:10.18632/oncotarget.20331

38. Mavrogenis AF, Megaloikonomos PD, Antoniadou T. Current concepts for the evaluation and management of diabetic foot ulcers. EFORT Open Rev. 2018;3(9):513-525. doi:10.1302/2058-5241.3.180010

39. Pemayun TG, Naibaho RM, Novitasari D. Risk factors for lower extremity amputation in patients with diabetic foot ulcers: a hospital-based case-control study. Diabet Foot Ankle. 2015;6(1):29629. doi:10.3402/dfa.v6.29629
40. Ochoa M Laser-processed parchment paper for fabrication of chronic wound dressings with selective oxygenation [Open Access Dissertations]. Purdue University; 2016:980.

41. Wu H, Li F, Shao W, et al. Promoting angiogenesis in oxidative diabetic wound microenvironment using a nanozyme-reinforced self-protecting hydrogel. ACS Cent Sci. 2019;5(3):477-485. doi:10. 1021/acscentsci.8b00850

\section{Publish your work in this journal}

The International Journal of Nanomedicine is an international, peerreviewed journal focusing on the application of nanotechnology in diagnostics, therapeutics, and drug delivery systems throughout the biomedical field. This journal is indexed on PubMed Central, MedLine, CAS, SciSearch ${ }^{\mathbb{B}}$, Current Contents ${ }^{\mathbb{B}} /$ Clinical Medicine, $^{2}$
Journal Citation Reports/Science Edition, EMBase, Scopus and the Elsevier Bibliographic databases. The manuscript management system is completely online and includes a very quick and fair peer-review system, which is all easy to use. Visit http://www.dovepress.com/ testimonials.php to read real quotes from published authors. 